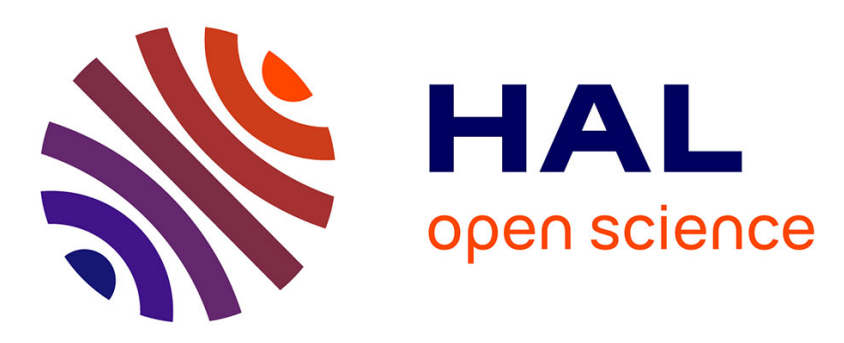

\title{
Cyclic Nucleotide Phosphodiesterases and Compartmentation in Normal and Diseased Heart
}

Ibrahim Bedioune, Pierre Bobin, Jérôme Leroy, Rodolphe Fischmeister, Grégoire Vandecasteele

\section{- To cite this version:}

Ibrahim Bedioune, Pierre Bobin, Jérôme Leroy, Rodolphe Fischmeister, Grégoire Vandecasteele. Cyclic Nucleotide Phosphodiesterases and Compartmentation in Normal and Diseased Heart. Viacheslav Nikolaev; Manuela Zaccolo. Microdomains in the Cardiovascular System, Springer, pp.97116, 2017, Cardiac and Vascular Biology, 978-3-319-54578-3. 10.1007/978-3-319-54579-0_6 . hal02472395

\section{HAL Id: hal-02472395 \\ https://hal.science/hal-02472395}

Submitted on 10 Feb 2020

HAL is a multi-disciplinary open access archive for the deposit and dissemination of scientific research documents, whether they are published or not. The documents may come from teaching and research institutions in France or abroad, or from public or private research centers.
L'archive ouverte pluridisciplinaire HAL, est destinée au dépôt et à la diffusion de documents scientifiques de niveau recherche, publiés ou non, émanant des établissements d'enseignement et de recherche français ou étrangers, des laboratoires publics ou privés. 


\title{
Cyclic nucleotide phosphodiesterases and compartmentation in normal and diseased heart
}

\author{
Ibrahim Bedioune, Pierre Bobin, Jérôme Leroy, \\ Rodolphe Fischmeister \& Grégoire Vandecasteele
}

UMR-S 1180, Inserm, Univ. Paris-Sud, Université Paris-Saclay, Châtenay-Malabry, France

\section{Correspondence:}

Grégoire Vandecasteele

UMR-S1180

Faculté de Pharmacie - Université Paris-Sud

5, Rue J.-B. Clément

F-92296 Châtenay-Malabry Cedex

France

Tel: 0146835717

Fax: 0146835475

gregoire.vandecasteele@u-psud.fr

OR

Rodolphe Fischmeister

UMR-S1180

Faculté de Pharmacie - Université Paris-Sud

5, Rue J.-B. Clément

F-92296 Châtenay-Malabry Cedex

France

Tel: 0146835771

Fax: 0146835475

rodolphe.fischmeister@inserm.fr 


\section{Abstract}

Cyclic nucleotide phosphodiesterases (PDEs) degrade the second messengers cAMP and cGMP, thereby regulating multiple aspects of cardiac function. This highly diverse class of enzymes encoded by twenty-one genes encompasses eleven families which are not only responsible for the termination of cyclic nucleotide signalling, but are also involved in the generation of dynamic microdomains of cAMP and cGMP controlling specific cell functions in response to various neurohormonal stimuli. In myocardium, the PDE3 and PDE4 families are predominant to degrade cAMP and thereby regulate cardiac excitation-contraction coupling. PDE3 inhibitors are positive inotropes and vasodilators in human, but their use is limited to acute heart failure and intermittent claudication. PDE5 is particularly important to degrade cGMP in vascular smooth muscle, and PDE5 inhibitors are used to treat erectile dysfunction and pulmonary hypertension. However, these drugs do not seem efficient in heart failure with preserved ejection fraction. There is experimental evidence that these PDEs as well as other PDE families including PDE1, PDE2 and PDE9 may play important roles in cardiac diseases such as hypertrophy and heart failure. After a brief presentation of the cyclic nucleotide pathways in cardiac cells and the major characteristics of the PDE superfamily, this chapter will present their role in cyclic nucleotide compartmentation and the current use of PDE inhibitors in cardiac diseases together with the recent research progresses that could lead to a better exploitation of the therapeutic potential of these enzymes in the future.

Keywords: cAMP, cGMP, cyclic nucleotide phosphodiesterases, heart failure

Abbreviations: AC, adenylyl cyclases; AKAP, A-kinase anchoring protein; ANP, atrial natriuretic peptide; $\beta$-ARs, $\beta$-adrenergic receptors; $\mathrm{BNP}$, brain natriuretic peptide; CaM, calmodulin; CaMKII, $\mathrm{Ca}^{2+} /$ Calmodulin-dependent kinase II; cAMP, cyclic adenosine monophosphate; cGMP, cyclic guanosine monophosphate; $\mathrm{CN}$, cyclic nucleotides; $\mathrm{CNP}, \mathrm{C}$ type natriuretic peptide; ECC, excitation-contraction coupling; Epac, exchange protein directly activated by cAMP; ERK, extracellular signal-regulated kinase; FRET, Förster resonance energy transfer; GAF, cGMP-stimulated phosphodiesterases, Anabaena adenylyl cyclases, Fhla transcription factor; GC, guanylyl cyclase; HF, heart failure; ICER, induciblecAMP early repressor; IR, ischemia-reperfusion; KO, knockout; LTCC, L-type $\mathrm{Ca}^{2+}$ channels; mAKAP, muscle AKAP; NO, nitric oxide; NOS, NO synthase; PDE, cyclic nucleotide phosphodiesterase; pGC, particulate guanylyl cyclase; PLB, phospholamban; PKA, cAMP- 
dependent protein kinase; PKG, cGMP-dependent protein kinase; PGE, prostaglandin; PI3K $\gamma$, phosphoinositide 3-kinase, $\gamma$ isoform; RyR2, ryanodine receptor type 2; SERCA, sarcoendoplasmic reticulum $\mathrm{Ca}^{2+}$-ATPase; sGC, soluble guanylyl cyclase; SR, sarcoplasmic reticulum; TnI, troponin $\mathrm{I}$. 


\section{Introduction}

The cyclic nucleotides $(\mathrm{CN})$ cAMP and cGMP participate in the main regulations of cardiac function. They act as second messengers for sympathetic and parasympathetic systems, nitric oxide (NO) and natriuretic peptides. $\mathrm{CN}$ may exert beneficial or deleterious effects on the heart, depending on the strength and duration of the stimulation. Acute elevation of $\mathrm{CN}$ regulates cardiac excitation-contraction coupling (ECC). However, chronic elevation of cAMP contributes to the development of cardiac hypertrophy and progression to heart failure (HF), while cGMP possesses anti-hypertrophic properties. The amplitude, duration and localization of $\mathrm{CN}$ responses are determined by the balance between synthesis of cAMP and cGMP by adenylyl and guanylyl cyclases, respectively, and degradation by cyclic nucleotide phosphodiesterases (PDEs). PDEs represent the main route to rapidly lower $\mathrm{CN}$ levels inside the cells, and constitute a highly diverse superfamily of enzymes. Different enzymatic properties and localization of multiple PDE isoforms within the cell participate in $\mathrm{CN}$ compartmentation, which is critical to determine specific physiological responses (Conti et al. 2014; Steinberg and Brunton 2001). In addition, modification in the expression and activity of specific PDEs are observed in several cardiovascular diseases. Thus, the members of the PDE superfamily are well placed to be the targets for pharmacological interventions in cardiovascular diseases. This is actually the case for a few of them, with PDE inhibitors being approved for the treatment of acute heart failure, erectile dysfunction, pulmonary hypertension, and intermittent claudication. In the following, we will present an overview of the roles of PDEs in cardiac muscle, the current indication of PDE inhibitors in heart diseases and the recent research advances holding promises for future therapeutic developments in cardiovascular diseases.

\section{CN signaling in cardiac myocytes}

In response to activation of $\mathrm{G}_{\mathrm{s}}$-coupled receptors, cAMP is produced by transmembrane adenylyl cyclases, which constitute the main source of cAMP in cardiac cells. Two types of guanylyl cyclases (GC) produce cGMP, the soluble GC (sGC) which is activated by NO, and particulate GCs (pGC) which constitute the receptors for natriuretic peptides (ANP, BNP and CNP). Once synthesized, $\mathrm{CN}$ exert their effects by acting through a limited number of cellular effectors: for cAMP, these include the cAMP-dependent protein kinase (PKA), cyclic nucleotide-gated ion channels (CNG), the exchange proteins directly activated by cAMP 
(Epac), and the recently discovered popeye-domain containing proteins (Schindler and Brand 2016). Similarly to cAMP, cGMP may exert its effects through activation of cGMPdependent protein kinase $(\mathrm{PKG})$ or $\mathrm{CNG}$ channels. Both nucleotides also bind directly to PDEs, not only at the catalytic site but also at allosteric sites, thereby modulating their activity.

During the fight or flight response, epinephrine and norepinephrine bind to $\beta$-adrenoceptors $(\beta$-ARs) in cardiomyocytes, leading to cAMP elevation and PKA activation. PKA phosphorylation of sarcolemmal L-type $\mathrm{Ca}^{2+}$ channels (LTCC), ryanodine receptors type 2 (RyR2), phospholamban (PLB, which controls the activity of the $\mathrm{Ca}^{2+}$-ATPase from the sarcoplasmic reticulum, SERCA2) and troponin I (TnI) enhances the amplitude and kinetics of $\mathrm{Ca}^{2+}$ transients in cardiomyocytes (Figure 1), underlying the classical positive inotropic and lusitropic effects of acute sympathetic stimulation. However, sustained stimulation of $\beta$-ARs, as occurs during hypertension or chronic heart diseases, is detrimental to the heart as it favors maladaptive hypertrophic remodeling, apoptosis and arrhythmias. Along with PKA, Epac is activated by cAMP and may play an important role in this context. Epac activation triggers a signaling pathway involving the phosphatase calcineurin and $\mathrm{Ca}^{2+} / \mathrm{Calmodulin}$-dependent kinase II (CaMKII) to stimulate hypertrophic growth (Lezoualc'h et al. 2016). CaMKII activation, which can also result from PKA-dependent increases in $\mathrm{Ca}^{2+}$, also phosphorylates RyR2 and promotes a pro-arrhythmogenic sarcoplasmic reticulum (SR) $\mathrm{Ca}^{2+}$ leak which may ultimately lead to chamber dilatation and HF (Ruiz-Hurtado et al. 2012) (Figure 1).

In the heart, cGMP is often viewed as the mirror of cAMP, opposing its effects on cardiac function. Indeed, cGMP can exert negative inotropic effects via PKG-mediated inhibition of the L-type $\mathrm{Ca}^{2+}$ current (Méry et al. 1991; Yang et al. 2007) and phosphorylation of TnI to decrease myofilament sensitivity to $\mathrm{Ca}^{2+}$ (Layland et al. 2005). In addition, cGMP can modulate cAMP levels through regulation of distinct PDEs (see below). One proposed mechanism by which cGMP-PKG signaling exerts its antihypertrophic action is by inhibiting the calcineurin pathway (Tsai and Kass 2009) (Figure 1).

\section{Overview of the PDE superfamily}

Eleven PDE families that differ in their primary structure, catalytic properties, affinities for cAMP and/or cGMP, as well as in their mechanisms of regulation are known in mammals (Figure 2). Most PDE families are encoded by several genes, which together generate close to 100 different PDE isoforms by the use of different translation initiation sites and alternative 
mRNA splicing. Specific isoforms are designated according to a common nomenclature: PDE is followed by a family number (1-11), a capital letter indicating the gene (A, B, C or D) and a final number corresponding to the splice variant. Some PDE families selectively hydrolyze cAMP (PDE4, 7, 8), while other are specific for cGMP (PDE5, 6, 9). A third category, so called dual PDEs, hydrolyzes both cAMP and cGMP (PDE1, 2, 3, 10, 11).

PDEs share a conserved catalytic domain (C-domain) showing approximately 25-52\% AA sequence identity, but differ markedly in their regulatory N-domain (Figure 2). N-domains contain diverse elements involved in enzyme dimerization, binding of regulatory small molecules, phosphorylation and localization. They are characteristic for each family and their variants. For instance, the unique distinguishing feature of the PDE1 family is the existence of two binding sites for the $\mathrm{Ca}^{2+}$-binding protein calmodulin $(\mathrm{CaM})$ in the $\mathrm{N}$-domain which are responsible for enzyme stimulation by $\mathrm{Ca}^{2+}$. Other important domains found in several PDE families (PDE2, PDE5, PDE6, PDE10 and PDE11) are the so-called GAF domains (this acronym is based on the first letters of the three proteins in which they were first identified: G: cGMP-stimulated phosphodiesterases; A: Anabaena adenylyl cyclase; F: Fhla transcription factor) which are involved in enzyme dimerization and allosteric regulation by cyclic nucleotides. In particular, in PDE2 and PDE5, cGMP-binding to their respective GAF domain stimulates enzymatic activity (Martins et al. 1982; Rybalkin et al. 2003). In contrast to PDE2, PDE3 is inhibited by cGMP, by direct competition at the catalytic site. The N-domains of various PDEs contain phosphorylation sites for distinct kinases which modulate enzymatic activity. For instance, long isoforms of PDE4 are phosphorylated by PKA, leading to an increase in cAMP-hydrolytic activity (Sette and Conti 1996), whereas PKG phosphorylation of PDE5 increases cGMP-hydrolytic activity (Francis et al. 2011). Long isoforms of PDE4D have been largely studied in that respect and were shown to be phosphorylated by multiple other kinases, including in particular ERK2, ERK5 and CaMKII (Mika et al. 2015). Ndomains are also important for intracellular localization, through specific regions that provide membrane association or protein-protein interaction. PDEs can associate with multiple protein partners including scaffold proteins such as A-kinase anchoring proteins (AKAPs) or $\beta$ arrestin strategically located within the cells. Recently, an interaction between PDE8 and the regulatory subunit RI $\alpha$ of PKA was described which brings together regions spanning the phosphodiesterase active site and cAMP-binding sites of RI $\alpha$ to facilitate cAMP hydrolysis (Krishnamurthy et al. 2014). More detailed presentations of PDEs including their structure, 
regulation, physiological roles and pharmacology are available in several recent reviews (Conti and Beavo 2007; Francis et al. 2011; Keravis and Lugnier 2012; Maurice et al. 2014).

\section{Role of PDEs in cyclic nucleotide compartmentation}

The notion of compartmentation arose from studies of cAMP signalling in the heart. Almost 40 years ago, Corbin and co-workers provided evidence that the two subtypes of PKA, designated type I and type II, are differentially distributed in the heart: while PKA type I was found mostly in the soluble fraction, PKA type II was predominant in the particulate fraction (Corbin et al. 1977). It was shown subsequently by Brunton and colleagues that the classical positive inotropic effect of $\beta$-AR stimulation was accompanied by an increase in cAMP and PKA in both fractions, whereas prostaglandin $\mathrm{E}_{1}\left(\mathrm{PGE}_{1}\right)$ stimulation increased cAMP and activated PKA only in the soluble fraction and was devoid of a positive inotropic effect (Buxton and Brunton 1983; Hayes et al. 1980). Two main conclusions were drawn from these experiments; the first was that cAMP generated by a given receptor does not activate all possible PKA molecules in the cell, and the second that active PKA cannot phosphorylate all possible substrates (Steinberg and Brunton 2001). The discovery of AKAPs as scaffold proteins able to direct PKA to specific subcellular compartments provided a mean to spatially restrict PKA activity (Dodge-Kafka et al. 2006; Wong and Scott 2004). A large number of PKA type II-specific AKAPs were shown to be associated with critical components of $\beta$-AR signalling, ECC or hypertrophic signalling in heart (for review see (Diviani et al. 2011)). The role of AKAPs in defining the range of PKA action is further supported by recent provocative experiments in HEK293 cells suggesting that when PKA is anchored to an AKAP, the catalytic subunits of the kinase may not dissociate from the regulatory subunits upon hormonal stimulation (Smith et al. 2013). Yet, these sophisticated assemblies of cAMP signalling components would not be sufficient to ensure specificity if cAMP can diffuse uniformly and indistinctly activate all PKAs present in a given cell. The observation that a local $\beta$-AR stimulation is much more efficient at stimulating local than remote $\mathrm{Ca}^{2+}$ channels in frog cardiomyocytes provided evidence that cAMP diffusion must be hindered. Importantly, global PDE inhibition with IBMX increased $\beta$-AR stimulation of the remote $\mathrm{Ca}^{2+}$ channels, implying that PDEs can act as a diffusion barrier preventing cAMP diffusion (Jurevicius and Fischmeister 1996). More direct evidence of the existence of cAMP gradients and extended cAMP spreading upon PDE inhibition were subsequently obtained by expressing cyclic nucleotide gated channels or Förster resonance energy transfer (FRET) 
biosensors to measure cAMP in real time in mammalian cardiomyocytes (Leroy et al. 2008; Molina et al. 2014; Mongillo et al. 2004; Nikolaev et al. 2006; Nikolaev et al. 2010; Zaccolo and Pozzan 2002). Based on experiments performed in HEK293 cells, it was proposed that differentially localized PDEs may function as local sinks, that drain cAMP concentration in defined domains by locally degrading the second messenger (Terrin et al. 2006). The interaction of PDE4D isoforms with several AKAPs facilitating PKA-mediated activation of the PDE by PKA also suggest that cAMP can be controlled locally, at the level of one macromolecular complex (Dodge et al. 2001; Terrenoire et al. 2009). Additional evidence that PDEs control local cAMP signals in cardiomyocytes come from studies showing that the contribution of the different PDEs to cAMP degradation depends on the type of $\mathrm{G}_{\mathrm{s}}$-coupled receptors generating the signal (Nikolaev et al. 2006; Rochais et al. 2006; Xiang et al. 2005). These studies highlighted the predominant role of the PDE4 family in this control, and in neonatal cardiac myocytes, distinct PDE4 variants were shown to associate with and regulate the cAMP signals generated by $\beta_{1}$-ARs versus $\beta_{2}$-ARs (Baillie et al. 2003; De Arcangelis et al. 2009; Mika et al. 2014; Richter et al. 2008; Richter et al. 2013). The use of cAMP biosensors specifically targeted to distinct subcellular compartments also suggests local cAMP degradation by distinct PDEs. For instance, targeting of FRET-based cAMP biosensors to the plasma membrane and the cytosol indicates a spatially restricted function of a specific PDE4 variant (PDE4B) to regulation of subsarcolemmal cAMP generated by $\beta_{1}$-ARs in neonatal cardiomyocytes (Mika et al. 2014). Moreover, cAMP biosensors targeted to PKA type I and II compartments are differentially activated by distinct receptors and PDE inhibitors: while cell stimulation with $\mathrm{PGE}_{1}$ or PDE2 inhibition preferentially increase cAMP in the PKA type I compartment, stimulation with Iso or PDE4 inhibition preferentially increase cAMP in the PKA type II compartment (Di Benedetto et al. 2008). Activation of PKA type II correlates with phosphorylation of SR proteins such as PLB by Iso, whereas $\mathrm{PGE}_{1} / \mathrm{E}_{2}$ fails to phosphorylate PLB (Di Benedetto et al. 2008; Liu et al. 2012). The use of a FRET-based PKA biosensor (AKAR) localized to the SR confirmed a lack of PKA activation in the SR upon $\mathrm{PGE}_{2}$ stimulation, but also showed that $\mathrm{PGE}_{2}$ is able to blunt $\beta$-AR inotropic response by activating PDE4D and preventing cAMP generated by $\beta$-ARs at the membrane to diffuse and activate PKA at the SR (Liu et al. 2012). Inhibition of PDE4 or ablation of the PDE4D gene were also shown to enhance PKA activation in the nucleus following a short stimulation of $\beta$-ARs, suggesting that PDE4D also impedes the diffusion of cAMP from the plasma membrane to the nucleus (Haj Slimane et al. 2014). 
While the works related above argue for a critical role of PDEs in cAMP compartmentation, a number of modelling studies suggest that PDEs are not sufficient to establish cAMP gradients if cAMP diffusion in the cell cytoplasm is as fast as in water (Feinstein et al. 2012; Iancu et al. 2007; Rich et al. 2001; Saucerman et al. 2014; Saucerman et al. 2006). Indeed, given their in vitro kinetics and estimated concentration in cells, PDEs may not be able to metabolize cAMP fast enough to avoid its replacement by diffusion from surrounding areas. Yet, at least for PDE2, there is convincing evidence that upon activation of the enzyme by cGMP, hydrolysis of cAMP is fast enough to balance the hormonal stimulation of cAMP synthesis (Fischmeister and Hartzell 1987; Hartzell and Fischmeister 1986; Nikolaev et al. 2005). Also, it should be kept in mind that PDE activity can be enhanced during hormonal stimulation (Conti et al. 2014). Whereas several studies indicated fast diffusion rates for cAMP in neurons (Bacskai et

al. 1993; Chen et al. 1999; Nikolaev et al. 2004), recent investigations suggest that cAMP diffusion is markedly slower in adult cardiomyocytes (Agarwal et al. 2016; Richards et al. 2016). The reasons for this are not entirely clear, but the above mentioned recent studies propose an important role of the mitochondrial network, acting either as a physical barrier (Richards et al. 2016) or as a support for anchored PKA acting as a cAMP buffer system (Agarwal et al. 2016).

\section{PDEs and cyclic nucleotide compartmentation in heart failure}

In the 1970s and 1980s, PDE3 inhibitors were discovered to exhibit cardiotonic, inotropic, bronchodilatory and vasodilatory activities in several species, and were initially developed as cardiotonic agents to replace or add to cardiac glycosides in the treatment of HF (Movsesian et al. 2011). However, despite beneficial hemodynamic effects on the short term, chronic use of PDE3 inhibitors were associated with increased cardiac arrhythmias and sudden death (Packer et al. 1991). Thus, the use of PDE3 inhibitors is now limited to acute decompensated HF. Nevertheless, PDE3 inhibitors are targeting several functionally distinct isoforms which are co-expressed in the heart, raising the hope that more selective targeting might provide some benefits. PDE3 is encoded by two genes, PDE3A and PDE3B. Evidence from global PDE3A and PDE3B knockout (KO) mice indicate that PDE3A but not PDE3B is responsible for the inotropic and chronotropic effects of PDE3 inhibitors (Sun et al. 2007). Three isoforms of PDE3A are expressed in cardiomyocytes, which differ only in their N-terminal domain, giving rise to different intracellular localization (Wechsler et al. 2002). In mice and humans, PDE3A1 controls PLB-SERCA2 activity and $\mathrm{Ca}^{2+}$ re-uptake in the SR (Ahmad et al. 2015; Beca et al. 2013) (Figure 1). Because dephosphorylated PLB and depressed SERCA2 activity 
are a hallmark of HF, PDE3 inhibitors targeting specifically the PDE3A1 associated with PLB-SERCA2 may improve contractile performance and provide therapy for HF (Movsesian 2015). However, currently available PDE3 inhibitors have little selectivity for PDE3A versus PDE3B isoforms, whose catalytic domains are similar, and no selectivity for individual PDE3A isoforms, whose catalytic domains are identical. Phosphorylation of PDE3A1 was recently shown to regulate its interaction with SERCA2 (Ahmad et al. 2015). Targeting this mechanism may offer an alternative to selectively enhance contractility without the harmful effects of global inhibition of PDE3 activity.

The second major PDE involved in cAMP hydrolysis in the heart is the cAMP-specific PDE4. The PDE4 family is encoded by 4 genes (PDE4A-D). Most of our knowledge on the role of individual PDE4 subtypes in the heart is limited to PDE4D. Deletion of this gene in mice leads to PKA hyperphosphorylation of RyR2, increased sensitivity to exercise-induced arrhythmias and a late onset dilated cardiomyopathy (Lehnart et al. 2005). PDE4D isoforms are localized in multiple compartments of the cardiomyocyte. For instance, PDE4D3 is localized at the perinuclear region, where it is part of a macromolecular complex organized by the scaffold protein mAKAP and comprising Epac1 and the kinase ERK5 to regulate cardiomyocyte hypertrophy (Dodge-Kafka et al. 2005). This isoform is also present at the sarcolemma, where it associates through another AKAP with slowly-activating delayed rectifier potassium channels controlling cardiac repolarization (Terrenoire et al. 2009) and at myofilaments, in association with another scaffold protein, myomegaline (Verde et al. 2001). In addition, as indicated above, distinct PDE4D isoforms were shown to interact with $\beta_{1}$-ARs and $\beta_{2}$-ARs, either directly or indirectly through $\beta$-arrestin, and to shape specific physiological or pathophysiological responses (Baillie et al. 2003; Berthouze-Duquesnes et al. 2013; De Arcangelis et al. 2009; Richter et al. 2008; Richter et al. 2013). Finally, similarly to PDE3A, PDE4D also associates with the PLB/SERCA2 complex and regulates the SERCA pump activity in the mouse heart (Beca et al. 2011) (Figure 1).

A role for PDE4B in the heart emerged recently when it was identified as an integral component of the LTCC complex and the main PDE regulating the LTCC current during $\beta$ AR stimulation (Figure 1). PDE4B KO mice, as PDE4D KO, have an increased susceptibility to ventricular arrhythmias during catecholamine stimulation which may be due to enhanced $\mathrm{Ca}^{2+}$ influx through LTCC (Leroy et al. 2011). Although RyR2 phosphorylation by PKA did not seem to be affected in adult hearts from PDE4B KO mice (Leroy et al. 2011), a recent study indicates that it was increased in neonatal myocytes lacking PDE4B (Mika et al. 2014) 
suggesting that altered RyR2 regulation may also contribute to this arrhythmic phenotype. In a recent study in rat ventricular myocytes, we showed that under $\beta$-AR stimulation, inhibition of PDE4 (as well as inhibition of PDE3) exerted inotropic effects via PKA but led to spontaneous diastolic $\mathrm{Ca}^{2+}$ waves via both PKA and CaMKII, suggesting the potential use of CaMKII inhibitors as adjuncts to PDE inhibition to limit their pro-arrhythmic effects (Bobin et al. 2016).

As stated above, phosphorylation of certain PDE3 and PDE4 isoforms by PKA activates these enzymes, and this constitutes a powerful negative feedback for cAMP signals in cardiomyocytes (Leroy et al. 2008; Rochais et al. 2004). This regulation has been shown to be facilitated by spatial proximity of PKA and PDEs assembled by the perinuclear mAKAP (Dodge-Kafka et al. 2005) or by PI3K $\gamma$, which in addition to its lipid kinase function also acts as an AKAP facilitating phosphorylation of PDE3B, PDE4A and PDE4B by PKA (Ghigo et al. 2012).

Although these studies underline the critical role of PDE4 in controlling $\beta$-AR stimulation in rodents, this family contributes less to the regulation of cardiac contractility in humans, where PDE3 predominates (Molenaar et al. 2013). However, in human atrial strips, inhibition of PDE3, but also of PDE4 potentiate the arrhythmogenic effect of $\beta$-AR stimulation, and PDE4 activity tends to decrease in the atria of patients with atrial fibrillation (Molina et al. 2012). A further understanding of the role of PDE4 in humans may also be important for the proarrhythmic effect of PDE3 inhibitors since PDE3 inhibitors such as milrinone and enoximone may also inhibit PDE4 in cardiac preparations (Bethke et al. 1992; Shakur et al. 2002).

In cardiac hypertrophy and HF, there are profound modifications in the major components of the cAMP pathway. These include a decreased density of $\beta_{1}$-ARs, an uncoupling of $\beta_{2}$-ARs from $G_{s}$, an increase in $G_{i}$ and in the G-protein coupled receptor kinase GRK2, and in certain models, a decrease in adenylyl cyclase activity (Lohse et al. 2003). In a model of pathological hypertrophy induced by pressure overload in rats, we found that the expression and activity of PDE3A, PDE4A and PDE4B were decreased, and this was associated with a blunted regulation of subsarcolemmal cAMP generated by $\beta$-ARs by PDE3 and PDE4 (Abi-Gerges et al. 2009). In contrast, in a model of cardiac hypertrophy induced by angiotensin II, an increased PDE4 activity was observed, accompanied by an increase in the 69-kDa-PDE4A isoform and a decrease in expression of 52- and 76-kDa PDE4D isoforms (Mokni et al. 2010). These results suggest that the level of expression of the isoforms of PDE3 and PDE4 is specifically regulated by the type of stimulus used to induce cardiac hypertrophy and the stage 
of the disease. Whereas an increase in cAMP-PDE can participate in desensitization of the $\beta$ AR pathway, a decrease could represent a compensatory mechanism to restore cAMP levels and inotropism. However, lower PDE activity also alters the degree of cAMP confinement, which could lead to illegitimate or excessive activation of certain pools of PKA or Epac, hence promoting maladaptive remodelling and rhythmic disturbances. This is supported by the results of a recent study showing that the specific PDE4D5 isoform regulates activation of hypertrophic program by Epac1 upon stimulation of $\beta_{2}$-AR (Berthouze-Duquesnes et al. 2013) receptors.

$\mathrm{HF}$ is also accompanied by modifications of the microarchitecture of the myocytes, including that of the T-tubular system, manifested as T-tubule loss or as reorganization depending on the studies (Louch et al. 2010). These alterations in the T-tubular network not only affect the synchrony of $\mathrm{Ca}^{2+}$ release (Heinzel et al. 2002), but are also associated with modifications in the distribution of $\mathrm{CN}$ signaling components and therefore, $\mathrm{CN}$ compartmentation. In a rat model of HF, it was shown that $\beta_{2}$-ARs redistribute from their normal T-tubular localization to the peripheral sarcolemma, leading to diffusive as opposed to constrained cAMP signals generated by these receptors (Nikolaev et al. 2010). In a more recent study, the local regulation of cAMP by PDEs in the vicinity of SERCA2 was compared in transgenic mice with cardiac-specific expression of a PLB-targeted cAMP biosensor, and modifications induced by transverse aortic constriction were analysed (Sprenger et al. 2015). In agreement with their known localization within the SERCA2 complex (Beca et al. 2013; Beca et al. 2011), both PDE3 and PDE4 were found to regulate cAMP in this microdomain. Interestingly, during hypertrophy and early HF, there was a specific rearrangement of the PDEs regulating this specific cAMP pool, with a decreased contribution of PDE4 and an increased contribution of PDE2 (Sprenger et al. 2015). These results indicate that PDE alterations in cardiac disease include redistribution of PDE variants in discrete microcompartments of cardiomyocytes, as shown for PDE2 ans PDE3 at the membrane (Perera et al. 2015).

The dual specific PDE2 represents a minor part of cAMP-hydrolytic activity in the normal heart, but the cAMP-hydrolytic activity of this PDE is stimulated 5 to 30 -fold by cGMP, and this was shown to inhibit cardiac LTCC in various species including humans (Fischmeister et al. 2005). Subsequently, measurements with FRET-based sensors in neonatal rat cardiomyocytes showed that by decreasing the level of cAMP, PDE2 counteracts the effects of a $\beta$-AR stimulation downstream of $\beta_{3}$-ARs (Mongillo et al. 2005). In contrast to PDE3 and 
PDE4, which expression and activity are generally decreased in pathological hypertrophy and HF (Abi-Gerges et al. 2009; Ding et al. 2005; Osadchii 2007), we found recently that PDE2 is increased in animal models as well as in human HF (Mehel et al. 2013). PDE2 inhibition partially restores $\beta$-AR responsiveness in diseased cardiomyocytes, suggesting that PDE2 enhancement in HF constitutes a protective mechanism against excessive $\beta$-AR stimulation. This hypothesis was confirmed in a more recent study using a transgenic mouse model with a cardiac-specific overexpression of PDE2 (Vettel et al. 2016). Transgenic increase in PDE2 abundance in mice lowers heart rate, but preserves cardiac output due to greater cardiac force. Increased PDE2 abundance was found to be cardioprotective in vivo in acute catecholaminergic stress and after myocardial infarction without compromising contractile performance (Vettel et al. 2016). However, according to another recent study, PDE2 could exert a pro-hypertrophic effect by blunting PKA-mediated phosphorylation of NFAT (Zoccarato et al. 2015). Further studies are needed to fully understand the role of PDE2 in HF (Wagner et al. 2016; Zoccarato et al. 2016).

Similarly to PDE2, PDE1 and PDE5 were reported to be upregulated in pathological hypertrophy and HF (Miller et al. 2009; Pokreisz et al. 2009; Vandeput et al. 2007). Because PDE1 and PDE5 preferentially (PDE1A) or specifically (PDE5) degrade cGMP, their increase in HF can clearly be seen as maladaptive. Accordingly, transgenic mice with cardiac-specific overexpression of PDE5 are predisposed to adverse remodelling after myocardial infarction (Pokreisz et al. 2009), whereas on the contrary, pharmacological inhibition of PDE1 (Miller et al. 2009) or PDE5 (Takimoto et al. 2005) reduces hypertrophy and improves cardiac pressure and volume overload. Numerous animal studies have shown that PDE5 inhibitors protect against ischemia/reperfusion (I/R) injury, doxorubicin cardiotoxicity, ischemic and diabetic cardiomyopathy, and Duchenne muscular dystrophy (Das et al. 2015). However, it remains controversial whether significant levels of PDE5 are expressed in the myocardium, raising the possibility that the beneficial effects of PDE5 inhibitors involve other mechanisms including inhibition of PDE1 (Degen et al. 2015; Lukowski et al. 2014). In patients with systolic HF, sildenafil decreased pulmonary vascular pressure and increased peak oxygen consumption and cardiac index (Lewis et al. 2007). Sildenafil also improved left ventricular diastolic function, cardiac geometry, and clinical status in patients with systolic HF (Guazzi et al. 2011) and improved diabetic cardiomyopathy (Giannetta et al. 2012). However, despite encouraging results in an initial mono-center study (Guazzi et al. 2011), chronic therapy with sildenafil was not associated with clinical benefit in patients with diastolic HF in a larger, 
multi-center study (Redfield et al. 2013). Ongoing trials with PDE5 inhibitors include testing for the gender response to tadalafil in left ventricular hypertrophy associated to diabetic cardiomyopathy (NCT01803828).

Two other PDEs were recently proposed to participate in cGMP degradation in the heart. Experiments performed in isolated cardiomyocytes from transgenic mice expressing a FRETbased cGMP biosensor have suggested that PDE3, which is classically known to degrade preferentially cAMP, may also be involved in the control of cGMP levels (Gotz et al. 2014). In addition, the cGMP-specific PDE9 was found to be expressed in rodent and human heart and to be up-regulated in hypertrophy and HF (Lee et al. 2015). PDE9 genetic ablation or pharmacological inhibition appears to protect the heart against pathological remodeling during pressure-overload. Moreover, PDE9 inhibition reverses pre-established heart disease in a NO synthase (NOS) activity independent manner, whereas PDE5 inhibition requires active NOS, which is decreased in HF. This is because PDE9 seems to hydrolyze specifically cGMP generated by natriuretic peptides, whereas PDE5 controls cGMP generated by NO (Castro et al. 2006; Takimoto et al. 2007). We had shown previously that PDE2 is critical to regulate subsarcolemmal cGMP levels in response to pGC activation in adult cardiomyocytes (Castro et al. 2006), thus raising the question of whether PDE2 and PDE9 exert a redundant or distinct regulation of natriuretic peptide signaling.

\section{PDEs as therapeutic targets in ischemia reperfusion injury?}

Manipulation of PDE activity may also prove protective in the context of ischemiareperfusion (IR) injury. Indeed, PDE5 inhibitors were shown to reduce infarct size in rabbits and mice. They also decreased cell death in isolated cardiomyocytes, suggesting that part of this effect is independent of vasodilation. Several mechanisms appear to be involved in these effects, including increased NO synthase expression, cGMP elevation, PKG activation and opening of mitochondrial $\mathrm{K}_{\mathrm{ATP}}$ and $\mathrm{Ca}^{2+}$-activated $\mathrm{K}^{+}$channels (Das et al. 2015). PDE3 inhibitors have also been reported to reduce infarct size when applied before sustained ischemia, thus mimicking the cardioprotection conferred by ischemic preconditioning (Fukasawa et al. 2008; Sanada et al. 2001; Tosaka et al. 2007). A recent study using KO mice for either PDE3A or PDE3B strongly suggests that PDE3B is the isoform mediating the cardioprotective effect of PDE3 inhibitors in this context. Indeed, PDE3B KO mice, but not PDE3A KO mice, were protected against ischemia-reperfusion injury. This protective effect appears to involve cAMP/PKA mediated opening of mitochondrial $\mathrm{Ca}^{2+}$-activated $\mathrm{K}^{+}$ 
channels and assembly of ischemia-induced caveolin 3 enriched fractions (Chung et al. 2015). Somehow at odds with the above mentioned cardioprotective effect of PDE3 inhibitors, mice with cardiac-specific overexpression of PDE3A1 were protected during ischemia-reperfusion injury (Oikawa et al. 2013). In addition to regulating SERCA2, PDE3A1 also acts as a negative regulator of cardiomyocyte apoptosis, by controlling the expression of the transcriptional repressor and pro-apoptotic factor, ICER (inducible- cAMP early repressor) (Yan et al. 2007). Inhibition of this mechanism in mice with cardiac-specific overexpression of PDE3A1 was associated with protection during ischemia-reperfusion (Oikawa et al. 2013). Collectively, these studies suggest that PDE3A and PDE3B may play an opposite role during ischemia-reperfusion, which may be linked to their differential localization and the control of discrete cAMP pools in cardiomyocytes (Chung et al. 2015).

\section{Concluding remarks}

Soon after the discovery of cAMP and cGMP by Sutherland and colleagues more than 50 years ago, an enzymatic activity that could degrade these second messengers was described (Sutherland and Rall 1958). Since then, the large diversity and complexity of the PDE superfamily has been unveiled, and the critical role of these enzymes in the cardiovascular system demonstrated. After several disappointments in the development of PDE3 and PDE4 inhibitors due to their adverse effects (mortality and emesis, respectively), the success in PDE5 inhibitors to treat erectile dysfunction associated with an increasing understanding of PDE biology has raised new hopes that manipulating PDE activity with greater specificity is possible and should yield therapeutic benefits. However, the targeting of individual PDE isoforms located in distinct subcellular compartments to regulate local cAMP and/or cGMP concentrations and a specific cell function is challenging. Knowledge of the protein partners and of the molecular mechanisms that govern this specific localization allows to envisage the displacement of specific PDE pools by small molecules or peptides that disrupt proteinprotein interaction, with potentially fewer adverse effects than global inhibition of an entire PDE family. Recent examples of such strategy include the disruption of PDE4 from heat shock protein 20 (HSP20) in cardiomyocytes, resulting in hyper-phosphorylation of HSP20 and attenuated cardiac hypertrophic growth (Martin et al. 2014; Sin et al. 2011) and the disruption of Epac1-PDE3B and Epac1-PDE4D complexes involved in the control of endothelial cell adhesion, spreading and permeability (Rampersad et al. 2010; Wilson et al. 2011). Another interesting strategy is the development of allosteric inhibitors of PDEs, for 
instance targeting the GAF domain of PDE5 to block enzyme activation but not its basal activity (Schultz et al. 2011) or exploiting sequence differences outside the active site to reach isoform selectivity in the case of PDE4B and PDE4D (Burgin et al. 2010; Fox et al. 2014). The allosteric mode of regulation could also allow the discovery of small molecules acting as activators of specific PDEs, which may actually be useful in correcting the detrimental effects of excessive $\beta$-AR stimulation observed in HF.

\section{Acknowledgements}

This work was supported by the Fondation de France (to GV) and the Agence Nationale de la Recherche 2010 BLAN 1139-01 (to GV). PB and IB were supported by PhD fellowships from the region Ile-de-France (CORDDIM) and Fondation pour la Recherche Médicale.

\section{Conflict of interest}

None. 


\section{References}

Abi-Gerges A, Richter W, Lefebvre F et al. (2009) Decreased expression and activity of cAMP phosphodiesterases in cardiac hypertrophy and its impact on $\beta$-adrenergic cAMP signals. Circ Res 105:784-792.

Agarwal SR, Clancy CE, Harvey RD (2016) Mechanisms restricting diffusion of intracellular cAMP. Sci Rep 6:19577.

Ahmad F, Shen W, Vandeput F et al. (2015) Regulation of sarcoplasmic reticulum $\mathrm{Ca}^{2+}$ ATPase 2 (SERCA2) activity by phosphodiesterase 3A (PDE3A) in human myocardium: Phosphorylation-dependent interaction of PDE3A1 with SERCA2. J Biol Chem 290:6763-76.

Bacskai BJ, Hochner B, Mahaut-Smith M et al. (1993) Spatially resolved dynamics of cAMP and protein kinase A subunits in Aplysia sensory neurons. Science 260:222-226.

Baillie GS, Sood A, McPhee I et al. (2003) $\beta$-Arrestin-mediated PDE4 cAMP phosphodiesterase recruitment regulates $\beta$-adrenoceptor switching from $G_{s}$ to $G_{i}$. Proc Natl Acad Sci USA 100:941-945.

Beca S, Ahmad F, Shen W et al. (2013) PDE3A regulates basal myocardial contractility through interacting with SERCA2a-signaling complexes in mouse heart. Circ Res 112:289-97.

Beca S, Helli PB, Simpson JA et al. (2011) Phosphodiesterase 4D regulates baseline sarcoplasmic reticulum $\mathrm{Ca}^{2+}$ release and cardiac contractility, independently of L-type $\mathrm{Ca}^{2+}$ current. Circ Res 109:1024-30.

Berthouze-Duquesnes M, Lucas A, Sauliere A et al. (2013) Specific interactions between Epac1, beta-arrestin2 and PDE4D5 regulate beta-adrenergic receptor subtypes differential effects on cardiac hypertrophic signaling. Cell Signal 25:970-80.

Bethke T, Eschenhagen T, Klimkiewicz A et al. (1992) Phosphodiesterase inhibition by enoximone in preparations from nonfailing and failing human hearts. Arzneimittelforschung 42:437-445.

Bobin P, Varin A, Lefebvre F, Fischmeister R, Vandecasteele G, Leroy J (2016) Calmodulin kinase II inhibition limits the pro-arrhythmic $\mathrm{Ca}^{2+}$ waves induced by cAMPphosphodiesterase inhibitors. Cardiovasc Res 110:151-61.

Burgin AB, Magnusson OT, Singh J et al. (2010) Design of phosphodiesterase 4D (PDE4D) allosteric modulators for enhancing cognition with improved safety. Nat Biotechnol 28:63-70.

Buxton ILO, Brunton LL (1983) Compartments of cyclic AMP and protein kinase in mammalian cardiomyocytes. J Biol Chem 258:10233-10239.

Castro LR, Verde I, Cooper DMF, Fischmeister R (2006) Cyclic guanosine monophosphate compartmentation in rat cardiac myocytes. Circulation 113:2221-2228.

Chen CH, Nakamura T, Koutalos Y (1999) Cyclic AMP diffusion coefficient in frog olfactory cilia. Biophys J 76:2861-2867.

Chung YW, Lagranha C, Chen Y et al. (2015) Targeted disruption of PDE3B, but not PDE3A, protects murine heart from ischemia/reperfusion injury. Proc Natl Acad Sci USA 112:E2253-62.

Conti M, Beavo JA (2007) Biochemistry and physiology of cyclic nucleotide phosphodiesterases: Essential components in cyclic nucleotide signaling. Ann Rev Biochem 76:481-511.

Conti M, Mika D, Richter W (2014) Perspectives on: Cyclic nucleotide microdomains and signaling specificity: Cyclic AMP compartments and signaling specificity: Role of cyclic nucleotide phosphodiesterases. J Gen Physiol 143:29-38. 
Corbin JD, Sugden PH, Lincoln TM, Keely SL (1977) Compartmentalization of adenosine 3':5'-monophosphate and adenosine 3':5'-monophosphate-dependent protein kinase in heart tissue. J Biol Chem 252:3854-3861.

Das A, Durrant D, Salloum FN, Xi L, Kukreja RC (2015) PDE5 inhibitors as therapeutics for heart disease, diabetes and cancer. Pharmacol Ther 147:12-21.

De Arcangelis V, Liu R, Soto D, Xiang Y (2009) Differential association of phosphodiesterase 4D isoforms with $\beta_{2}$-adrenoceptor in cardiac myocytes. $\mathrm{J}$ Biol Chem 284:33824-32.

Degen CV, Bishu K, Zakeri R, Ogut O, Redfield MM, Brozovich FV (2015) The Emperor's New Clothes: PDE5 and the Heart. PLoS One 10:e0118664.

Di Benedetto G, Zoccarato A, Lissandron V et al. (2008) Protein kinase A type I and type II define distinct intracellular signaling compartments. Circ Res 103:836-44.

Ding B, Abe J, Wei H et al. (2005) A positive feedback loop of phosphodiesterase 3 (PDE3) and inducible cAMP early repressor (ICER) leads to cardiomyocyte apoptosis. Proc Natl Acad Sci USA 102:14771-14776.

Diviani D, L Dodge-Kafka K, Li J, Kapiloff MS (2011) A-kinase anchoring proteins: Scaffolding proteins in the heart. Am J Physiol Heart Circ Physiol 301:H1742-H1753.

Dodge-Kafka KL, Langeberg L, Scott JD (2006) Compartmentation of cyclic nucleotide signaling in the heart: the role of A-kinase anchoring proteins. Circ Res 98:993-1001.

Dodge-Kafka KL, Soughayer J, Pare GC et al. (2005) The protein kinase A anchoring protein mAKAP co-ordinates two integrated cAMP effector pathways. Nature 437:574-578.

Dodge KL, Khouangsathiene S, Kapiloff MS et al. (2001) mAKAP assembles a protein kinase A/PDE4 phosphodiesterase cAMP signaling module. EMBO J 20:1921-1930.

Feinstein WP, Zhu B, Leavesley SJ, Sayner SL, Rich TC (2012) Assessment of cellular mechanisms contributing to cAMP compartmentalization in pulmonary microvascular endothelial cells. Am J Physiol Cell Physiol 302:C839-52.

Fischmeister R, Castro L, Abi-Gerges A, Rochais F, Vandecasteele G (2005) Species-and tissue-dependent effects of NO and cyclic GMP on cardiac ion channels. Comp Biochem Physiol A Mol Integr Physiol 142:136-143.

Fischmeister R, Hartzell HC (1987) Cyclic guanosine 3',5'-monophosphate regulates the calcium current in single cells from frog ventricle. J Physiol 387:453-472.

Fox D, 3rd, Burgin AB, Gurney ME (2014) Structural basis for the design of selective phosphodiesterase 4B inhibitors. Cell Signal 26:657-63.

Francis SH, Blount MA, Corbin JD (2011) Mammalian cyclic nucleotide phosphodiesterases: molecular mechanisms and physiological functions. Physiol Rev 91:651-90.

Fukasawa M, Nishida H, Sato T, Miyazaki M, Nakaya H (2008) 6-[4-(1-Cyclohexyl-1Htetrazol-5-yl)butoxy]-3,4-dihydro-2-(1H)quinolinone (cilostazol), a phosphodiesterase type 3 inhibitor, reduces infarct size via activation of mitochondrial $\mathrm{Ca}^{2+}$-activated $\mathrm{K}^{+}$ channels in rabbit hearts. J Pharmacol Exp Ther 326:100-4.

Ghigo A, Perino A, Mehel H et al. (2012) PI3KY Protects against catecholamine-induced ventricular arrhythmia through PKA-mediated regulation of distinct phosphodiesterases. Circulation 126:2073-2083.

Giannetta E, Isidori AM, Galea N et al. (2012) Chronic Inhibition of cGMP phosphodiesterase 5A improves diabetic cardiomyopathy: a randomized, controlled clinical trial using magnetic resonance imaging with myocardial tagging. Circulation 125:2323-33.

Gotz K, Sprenger J, Perera RK et al. (2014) Transgenic mice for real time visualization of cGMP in intact adult cardiomyocytes. Circ Res 114:1235-45.

Guazzi M, Vicenzi M, Arena R, Guazzi MD (2011) PDE5 inhibition with sildenafil improves left ventricular diastolic function, cardiac geometry, and clinical status in patients with 
stable systolic heart failure: results of a 1-year, prospective, randomized, placebocontrolled study. Circ Heart Fail 4:8-17.

Haj Slimane Z, Bedioune I, Lechêne P et al. (2014) Control of cytoplasmic and nuclear protein kinase A activity by phosphodiesterases and phosphatases in cardiac myocytes. Cardiovasc Res 102:97-106.

Hartzell HC, Fischmeister R (1986) Opposite effects of cyclic GMP and cyclic AMP on $\mathrm{Ca}^{2+}$ current in single heart cells. Nature 323:273-275.

Hayes JS, Brunton LL, Mayer SE (1980) Selective activation of particulate cAMP-dependent protein kinase by isoproterenol and prostaglandin E1. J Biol Chem 255:5113-5119.

Heinzel FR, Bito V, Volders PG, Antoons G, Mubagwa K, Sipido KR (2002) Spatial and temporal inhomogeneities during $\mathrm{Ca}^{2+}$ release from the sarcoplasmic reticulum in pig ventricular myocytes. Circ Res 91:1023-30.

Iancu RV, Jones SW, Harvey RD (2007) Compartmentation of cAMP signaling in cardiac myocytes: A computational study. Biophys J 92:3317-3331.

Jurevicius J, Fischmeister R (1996) cAMP compartmentation is responsible for a local activation of cardiac $\mathrm{Ca}^{2+}$ channels by $\beta$-adrenergic agonists. Proc Natl Acad Sci USA 93:295-299.

Keravis T, Lugnier C (2012) Cyclic nucleotide phosphodiesterase (PDE) isozymes as targets of the intracellular signalling network: benefits of PDE inhibitors in various diseases and perspectives for future therapeutic developments. Br J Pharmacol 165:1288-305.

Krishnamurthy S, Moorthy BS, Xin Xiang L et al. (2014) Active site coupling in PDE:PkA complexes promotes resetting of mammalian cAMP signaling. Biophys J 107:142640.

Layland J, Solaro RJ, Shah AM (2005) Regulation of cardiac contractile function by troponin I phosphorylation. Cardiovasc Res 66:12-21.

Lee DI, Zhu G, Sasaki T et al. (2015) Phosphodiesterase 9A controls nitric-oxide-independent cGMP and hypertrophic heart disease. Nature 519:472-6.

Lehnart SE, Wehrens XHT, Reiken S et al. (2005) Phosphodiesterase 4D deficiency in the ryanodine receptor complex promotes heart failure and arrhythmias. Cell 123:23-35.

Leroy J, Abi-Gerges A, Nikolaev VO et al. (2008) Spatiotemporal dynamics of B-adrenergic cAMP signals and L-type $\mathrm{Ca}^{2+}$ channel regulation in adult rat ventricular myocytes: Role of phosphodiesterases. Circ Res 102:1091-1100.

Leroy J, Richter W, Mika D et al. (2011) Phosphodiesterase 4B in the cardiac L-type $\mathrm{Ca}^{2+}$ channel complex regulates $\mathrm{Ca}^{2+}$ current and protects against ventricular arrhythmias. $\mathrm{J}$ Clin Invest 121:2651-61.

Lewis GD, Lachmann J, Camuso J et al. (2007) Sildenafil improves exercise hemodynamics and oxygen uptake in patients with systolic heart failure. Circulation 115:59-66.

Lezoualc'h F, Fazal L, Laudette M, Conte C (2016) Cyclic AMP sensor EPAC proteins and their role in cardiovascular function and disease. Circ Res 118:881-97.

Liu S, Li Y, Kim S et al. (2012) Phosphodiesterases coordinate cAMP propagation induced by two stimulatory G protein-coupled receptors in hearts. Proc Natl Acad Sci USA 109:6578-83.

Lohse MJ, Engelhardt S, Eschenhagen T (2003) What is the role of $B$-adrenergic signaling in heart failure? Circ Res 93:896-906.

Louch WE, Hake J, Jolle GF et al. (2010) Control of $\mathrm{Ca}^{2+}$ release by action potential configuration in normal and failing murine cardiomyocytes. Biophys J 99:1377-86.

Lukowski R, Krieg T, Rybalkin SD, Beavo J, Hofmann F (2014) Turning on cGMPdependent pathways to treat cardiac dysfunctions: boom, bust, and beyond. Trends Pharmacol Sci 35:404-413. 
Martin TP, Hortigon-Vinagre MP, Findlay JE, Elliott C, Currie S, Baillie GS (2014) Targeted disruption of the heat shock protein 20-phosphodiesterase 4D (PDE4D) interaction protects against pathological cardiac remodelling in a mouse model of hypertrophy. FEBS Open Bio 4:923-927.

Martins TJ, Mimby MC, Beavo JA (1982) Purification and characterization of a cyclic GMPstimulated cyclic nucleotide phosphodiesterase from bovine tissues. J Biol Chem 257:1973-1979.

Maurice DH, Ke H, Ahmad F, Wang Y, Chung J, Manganiello VC (2014) Advances in targeting cyclic nucleotide phosphodiesterases. Nat Rev Drug Discov 13:290-314.

Mehel H, Emons J, Vettel C et al. (2013) Phoshodiesterase-2 is upregulated in human failing hearts and blunts $B$-adrenergic responses in cardiomyocytes. J Am Coll Cardiol 62:1596-1606.

Méry P-F, Lohmann SM, Walter U, Fischmeister R (1991) $\mathrm{Ca}^{2+}$ current is regulated by cyclic GMP-dependent protein kinase in mammalian cardiac myocytes. Proc Natl Acad Sci USA 88:1197-1201.

Mika D, Richter W, Conti M (2015) A CaMKII/PDE4D negative feedback regulates cAMP signaling. Proc Natl Acad Sci USA 112:2023-8.

Mika D, Richter W, Westenbroek RE, Catterall WA, Conti M (2014) PDE4B mediates local feedback regulation of $\beta_{1}$-adrenergic cAMP signaling in a sarcolemmal compartment of cardiac myocytes. J Cell Sci 127:1033-1042.

Miller CL, Oikawa M, Cai Y et al. (2009) Role of $\mathrm{Ca}^{2+} /$ calmodulin-stimulated cyclic nucleotide phosphodiesterase 1 in mediating cardiomyocyte hypertrophy. Circ Res 105:956-64.

Mokni W, Keravis T, Etienne-Selloum N et al. (2010) Concerted regulation of cGMP and cAMP phosphodiesterases in early cardiac hypertrophy induced by angiotensin II. PLoS One 5:e14227.

Molenaar P, Christ T, Hussain RI et al. (2013) Phosphodiesterase PDE3, but not PDE4, reduces $\beta_{1}$ - and $\beta_{2}$-adrenoceptor-mediated inotropic and lusitropic effects in failing ventricle from metoprolol-treated patients. Br J Pharmacol 169:528-538.

Molina CE, Johnson DM, Mehel $\mathrm{H}$ et al. (2014) Interventricular differences in B-adrenergic responses in the canine heart: Role of phosphodiesterases. J Am Heart Ass (JAHA) 3:pii: e000858. doi: 10.1161/JAHA.114.000858.

Molina CE, Leroy J, Xie M et al. (2012) Cyclic AMP phosphodiesterase type 4 protects against atrial arrhythmias. J Am Coll Cardiol 59:2182-2190.

Mongillo M, McSorley T, Evellin S et al. (2004) Fluorescence resonance energy transferbased analysis of cAMP dynamics in live neonatal rat cardiac myocytes reveals distinct functions of compartmentalized phosphodiesterases. Circ Res 95:65-75.

Mongillo M, Terrin A, Evellin S, Lissandron V, Zaccolo M (2005) Study of cyclic adenosine monophosphate microdomains in cells. Meth Mol Biol 307:1-13.

Movsesian M (2015) New pharmacologic interventions to increase cardiac contractility: challenges and opportunities. Curr Opin Cardiol:(in press).

Movsesian M, Wever-Pinzon O, Vandeput F (2011) PDE3 inhibition in dilated cardiomyopathy. Curr Opin Pharmacol.

Nikolaev VO, Bunemann M, Hein L, Hannawacker A, Lohse MJ (2004) Novel single chain cAMP sensors for receptor-induced signal propagation. J Biol Chem 279:3721537218.

Nikolaev VO, Bunemann M, Schmitteckert E, Lohse MJ, Engelhardt S (2006) Cyclic AMP imaging in adult cardiac myocytes reveals far-reaching $\beta_{1}$-adrenergic but locally confined $\beta_{2}$-adrenergic receptor-mediated signaling. Circ Res 99:1084-1091. 
Nikolaev VO, Gambaryan S, Engelhardt S, Walter U, Lohse MJ (2005) Real-time monitoring of live cell's PDE2 activity: Hormone-stimulated cAMP hydrolysis is faster than hormone-stimulated cAMP synthesis. J Biol Chem 280:1716-1719.

Nikolaev VO, Gambaryan S, Lohse MJ (2006) Fluorescent sensors for rapid monitoring of intracellular cGMP. Nat Methods 3:23-25.

Nikolaev VO, Moshkov A, Lyon AR et al. (2010) $\beta_{2}$-Adrenergic receptor redistribution in heart failure changes cAMP compartmentation. Science 327:1653-7.

Oikawa M, Wu M, Lim S et al. (2013) Cyclic nucleotide phosphodiesterase 3A1 protects the heart against ischemia-reperfusion injury. J Mol Cell Cardiol 64:11-19.

Osadchii OE (2007) Myocardial phosphodiesterases and regulation of cardiac contractility in health and cardiac disease. Cardiovasc Drugs Ther 21:171-94.

Packer M, Carver JR, Rodeheffer RJ et al. (1991) Effect of oral milrinone on mortality in severe chronic heart failure. The PROMISE Study Research Group. N Engl J Med 325:1468-1475.

Perera RK, Sprenger JU, Steinbrecher JH et al. (2015) Microdomain switch of cGMPregulated phosphodiesterases leads to ANP-induced augmentation of betaadrenoceptor-stimulated contractility in early cardiac hypertrophy. Circ Res 116:130411.

Pokreisz P, Vandenwijngaert S, Bito V et al. (2009) Ventricular phosphodiesterase-5 expression is increased in patients with advanced heart failure and contributes to adverse ventricular remodeling after myocardial infarction in mice. Circulation 119:408-416.

Rampersad SN, Ovens JD, Houston E et al. (2010) Cyclic AMP phosphodiesterase 4D (PDE4D) tethers EPAC1 in a VE-cadherin (VECAD)-based signaling complex and controls cAMP-mediated vascular permeability. J Biol Chem 285:33614-22.

Redfield MM, Chen HH, Borlaug BA et al. (2013) Effect of phosphodiesterase-5 inhibition on exercise capacity and clinical status in heart failure with preserved ejection fraction: a randomized clinical trial. JAMA 309:1268-77.

Rich TC, Fagan KA, Tse TE, Schaack J, Cooper DM, Karpen JW (2001) A uniform extracellular stimulus triggers distinct cAMP signals in different compartments of a simple cell. Proc Natl Acad Sci USA 98:13049-13054.

Richards M, Lomas O, Jalink K et al. (2016) Intracellular tortuosity underlies slow cAMP diffusion in adult ventricular myocytes. Cardiovasc Res 110:395-407.

Richter W, Day P, Agraval R et al. (2008) Signaling from $\beta_{1}$-and $\beta_{2}$-adrenergic receptors is defined by differential interactions with PDE4. Embo J 27:384-393.

Richter W, Mika D, Blanchard E, Day P, Conti M (2013) $\beta_{1}$-adrenergic receptor antagonists signal via PDE4 translocation. EMBO Reports 14:276-83.

Rochais F, Abi-Gerges A, Horner K et al. (2006) A specific pattern of phosphodiesterases controls the cAMP signals generated by different $\mathrm{G}_{\mathrm{s}}$-coupled receptors in adult rat ventricular myocytes. Circ Res 98:1081-1088.

Rochais F, Vandecasteele G, Lefebvre F et al. (2004) Negative feedback exerted by PKA and cAMP phosphodiesterase on subsarcolemmal cAMP signals in intact cardiac myocytes. An in vivo study using adenovirus-mediated expression of CNG channels. $\mathbf{J}$ Biol Chem 279:52095-52105.

Ruiz-Hurtado G, Dominguez-Rodriguez A, Pereira L et al. (2012) Sustained Epac activation induces calmodulin dependent positive inotropic effect in adult cardiomyocytes. J Mol Cell Cardiol 53:617-25.

Rybalkin SD, Rybalkina IG, Shimizu-Albergine M, Tang XB, Beavo JA (2003) PDE5 is converted to an activated state upon cGMP binding to the GAF A domain. EMBO J 22:469-78. 
Sanada S, Kitakaze M, Papst PJ et al. (2001) Cardioprotective effect afforded by transient exposure to phosphodiesterase III inhibitors -The role of protein kinase A and p38 mitogen-activated protein kinase. Circulation 104:705-710.

Saucerman JJ, Greenwald EC, Polanowska-Grabowska R (2014) Perspectives on: Cyclic nucleotide microdomains and signaling specificity: Mechanisms of cyclic AMP compartmentation revealed by computational models. J Gen Physiol 143:39-48.

Saucerman JJ, Zhang J, Martin JC et al. (2006) Systems analysis of PKA-mediated phosphorylation gradients in live cardiac myocytes. Proc Natl Acad Sci USA 103:12923-12928.

Schindler RF, Brand T (2016) The Popeye domain containing protein family--A novel class of cAMP effectors with important functions in multiple tissues. Prog Biophys Mol Biol 120:28-36.

Schultz JE, Dunkern T, Gawlitta-Gorka E, Sorg G (2011) The GAF-tandem domain of phosphodiesterase 5 as a potential drug target. Handb Exp Pharmacol:151-66.

Sette C, Conti M (1996) Phosphorylation and activation of a cAMP-specific phosphodiesterase by the cAMP-dependent protein kinase -Involvement of serine 54 in the enzyme activation. J Biol Chem 271:16526-16534.

Shakur Y, Fong M, Hensley J et al. (2002) Comparison of the effects of cilostazol and milrinone on cAMP-PDE activity, intracellular cAMP and calcium in the heart. Cardiovasc Drugs Ther 16:417-427.

Sin YY, Edwards HV, Li X et al. (2011) Disruption of the cyclic AMP phosphodiesterase-4 (PDE4)-HSP20 complex attenuates the beta-agonist induced hypertrophic response in cardiac myocytes. J Mol Cell Cardiol 50:872-83.

Smith FD, Reichow SL, Esseltine JL et al. (2013) Intrinsic disorder within an AKAP-protein kinase A complex guides local substrate phosphorylation. Elife 2:e01319.

Sprenger JU, Perera RK, Steinbrecher JH et al. (2015) In vivo model with targeted cAMP biosensor reveals changes in receptor-microdomain communication in cardiac disease. Nat Commun 6:6965.

Steinberg SF, Brunton LL (2001) Compartmentation of G protein-coupled signaling pathways in cardiac myocytes. Ann Rev Pharmacol Toxicol 41:751-773.

Sun B, Li H, Shakur Y et al. (2007) Role of phosphodiesterase type 3A and 3B in regulating platelet and cardiac function using subtype-selective knockout mice. Cell Signal 19:1765-71.

Sutherland EW, Rall TW (1958) Fractionation and characterization of a cyclic adenine ribonucleotide formed by tissue particles. J Biol Chem 232:1077-1091.

Takimoto E, Belardi D, Tocchetti CG et al. (2007) Compartmentalization of cardiac Badrenergic inotropy modulation by phosphodiesterase type 5. Circulation 115:215967.

Takimoto E, Champion HC, Li M et al. (2005) Chronic inhibition of cyclic GMP phosphodiesterase 5A prevents and reverses cardiac hypertrophy. Nat Med 11:214222.

Terrenoire C, Houslay MD, Baillie GS, Kass RS (2009) The cardiac IKs potassium channel macromolecular complex includes the phosphodiesterase PDE4D3. J Biol Chem 284:9140-6.

Terrin A, Di Benedetto G, Pertegato V et al. (2006) PGE1 stimulation of HEK293 cells generates multiple contiguous domains with different [cAMP]: role of compartmentalized phosphodiesterases. J Cell Biol 175:441-51.

Tosaka S, Makita T, Tosaka R et al. (2007) Cardioprotection induced by olprinone, a phosphodiesterase III inhibitor, involves phosphatidylinositol-3-OH kinase-Akt and a 
mitochondrial permeability transition pore during early reperfusion. J Anesth 21:17680.

Tsai EJ, Kass DA (2009) Cyclic GMP signaling in cardiovascular pathophysiology and therapeutics. Pharmacol Ther 122:216-38.

Vandeput F, Wolda SL, Krall J et al. (2007) Cyclic nucleotide phosphodiesterase PDE1C in human cardiac myocytes. J Biol Chem 282:32749-57.

Verde I, Pahlke G, Salanova M et al. (2001) Myomegalin is a novel protein of the Golgi/centrosome that interacts with a cyclic nucleotide phosphodiesterase. J Biol Chem 276:11189-11198.

Vettel C, Lindner M, Dewenter M et al. (2016) Phosphodiesterase 2 protects against catecholamine-induced arrhythmias and preserves contractile function after myocardial infarction. Circ Res:(in press).

Wagner M, Mehel H, Fischmeister R, El-Armouche A (2016) Phosphodiesterase 2: antiadrenergic friend or hypertrophic foe in heart disease? Naunyn Schmiedebergs Arch Pharmacol:(in press).

Wechsler J, Choi YH, Krall J, Ahmad F, Manganiello VC, Movsesian MA (2002) Isoforms of cyclic nucleotide phosphodiesterase PDE3A in cardiac myocytes. J Biol Chem 277:38072-38078.

Wilson LS, Baillie GS, Pritchard LM et al. (2011) A phosphodiesterase 3B-based signaling complex integrates exchange protein activated by cAMP 1 and phosphatidylinositol 3kinase signals in human arterial endothelial cells. J Biol Chem 286:16285-96.

Wong W, Scott JD (2004) AKAP signalling complexes: focal points in space and time. Nat Rev Mol Cell Biol 5:959-970.

Xiang Y, Naro F, Zoudilova M, Jin SL, Conti M, Kobilka B (2005) Phosphodiesterase 4D is required for $\beta_{2}$ adrenoceptor subtype-specific signaling in cardiac myocytes. Proc Natl Acad Sci USA 102:909-914.

Yan C, Miller CL, Abe J (2007) Regulation of phosphodiesterase 3 and inducible cAMP early repressor in the heart. Circ Res 100:489-501.

Yang L, Liu G, Zakharov SI, Bellinger AM, Mongillo M, Marx SO (2007) Protein kinase G phosphorylates Cav1.2 alpha1c and beta2 subunits. Circ Res 101:465-74.

Zaccolo M, Pozzan T (2002) Discrete microdomains with high concentration of cAMP in stimulated rat neonatal cardiac myocytes. Science 295:1711-1715.

Zoccarato A, Fields LH, Zaccolo M (2016) Response to Wagner et al.: phosphodiesterase-2anti-adrenergic friend or hypertrophic foe in heart disease? Naunyn Schmiedebergs Arch Pharmacol:(in press).

Zoccarato A, Surdo NC, Aronsen JM et al. (2015) Cardiac hypertrophy is inhibited by a local pool of cAMP regulated by phosphodiesterase 2. Circ Res 117:707-19. 


\section{Figure legends}

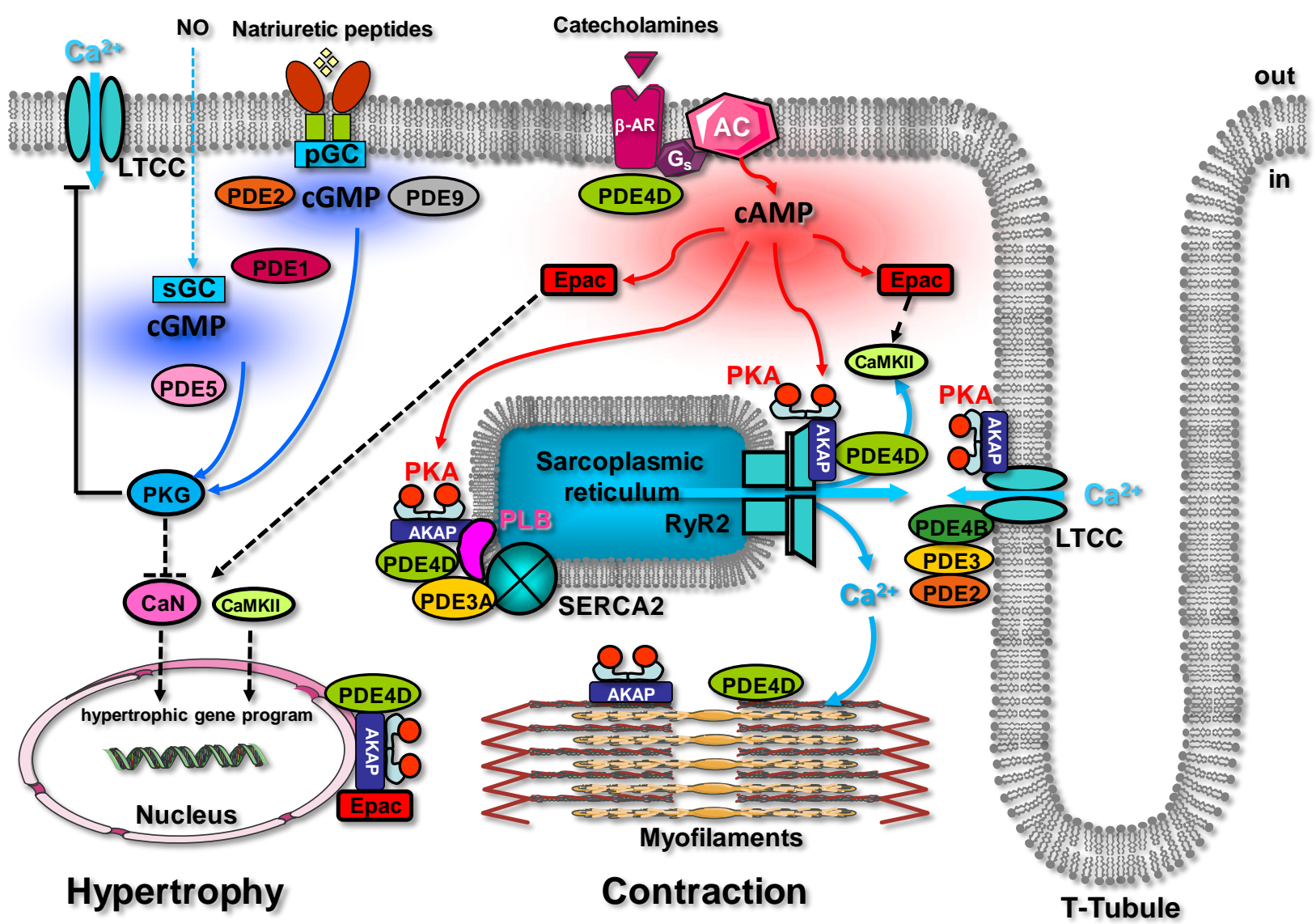

Figure 1: Cyclic nucleotides metabolism in cardiac myocytes.

The major PDEs expressed in cardiac myocytes are indicated together with their subcellular localization in relation to their role in regulating hypertrophic growth and excitationcontraction coupling. For simplicity, the PDE family and - when relevant - the gene are indicated but not the specific splice variants. AC, adenylyl cyclase; AKAP, A-kinase anchoring protein; CaMKII, $\mathrm{Ca}^{2+}$-Calmodulin kinase II; CaN, calcineurin; GPCR, G-protein coupled receptor; pGC, particulate guanylyl cyclase; sGC, soluble guanylyl cyclase. 

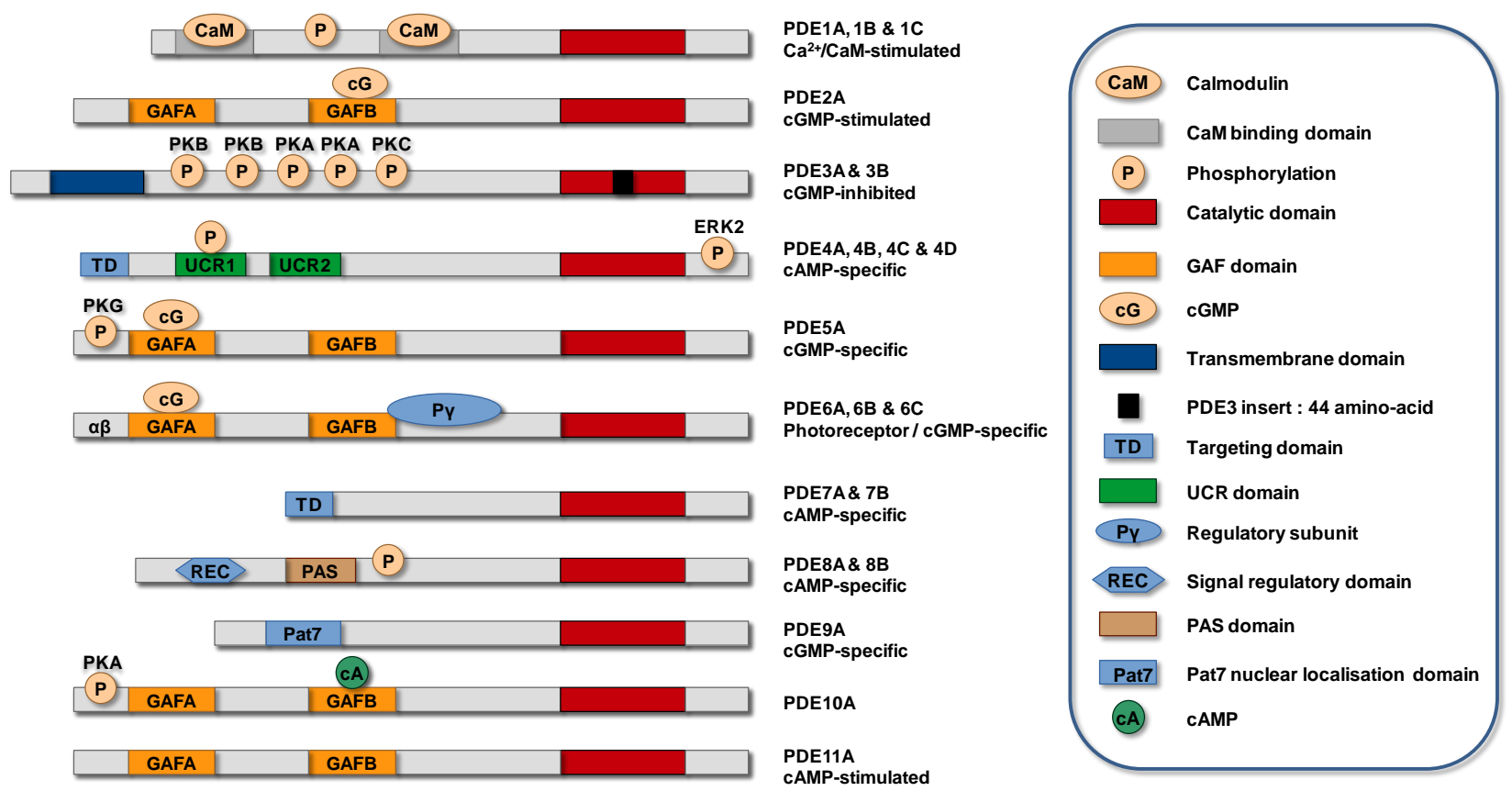

Figure 2: The eleven mammalian PDE families. The conserved catalytic domain (shown in red) is located in the carboxy-terminal portion of the PDEs. The catalytic domain of PDE3 contains a unique 44-amino-acid insert (shown in black). Many of the PDE families contain amino-terminal subdomains (such as GAF domains, transmembrane domains, targeting domains, upstream conserved regions (UCRs), PAS domains and REC domains) and Nterminal hydrophobic regions that are important in subcellular localization, in the incorporation of PDEs into compartmentalized signalosomes, in interactions with signalling molecules and molecular scaffolds, and in the regulation of PDE activity. GAF domains regulate the allosteric binding of cGMP (to PDE2, PDE5, PDE6 and PDE11), the allosteric binding of cAMP (to PDE10) and the regulation of catalytic activity (in PDE2, PDE5 and PDE6). (Modified from ref.(Maurice et al. 2014)) 